Résumés des conférences et travaux

\title{
Histoire et philologie de la Mésopotamie
}

\section{Michaël Guichard}

\section{OpenEdition \\ Journals}

\section{Édition électronique}

URL : https://journals.openedition.org/ashp/4110

DOI : $10.4000 /$ ashp. 4110

ISSN : 1969-6310

\section{Éditeur}

Publications de l'École Pratique des Hautes Études

\section{Édition imprimée}

Date de publication : 1 septembre 2021

Pagination : $32-45$

ISSN : 0766-0677

\section{Référence électronique}

Michaël Guichard, "Histoire et philologie de la Mésopotamie », Annuaire de l'École pratique des hautes études (EPHE), Section des sciences historiques et philologiques [En ligne], 152 | 2021, mis en ligne le 14 juin 2021, consulté le 13 juin 2022. URL : http://journals.openedition.org/ashp/4110 ; DOl : https:// doi.org/10.4000/ashp.4110 


\title{
HISTOIRE ET PHILOLOGIE DE LA MÉSOPOTAMIE
}

\author{
Directeur d'études : M. Michaël GuichaRD
}

Programme de l'année 2019-2020: Histoire et littérature : étude d'Innana et la montagne d'Ebih (épigraphie sumérienne).

L'incipit Maîtresse aux dangereux pouvoirs (cosmiques) (in-nin(g)-me-huš-a) désigne une composition sumérienne intitulée dans la littérature moderne Innana et (l')Ebih. C'est un des classiques de la culture mésopotamienne. Il en était déjà ainsi d'une certaine façon au début du deuxième millénaire puisque le texte a été copié par des générations de scribes sur une durée d'au moins deux siècles. On peut estimer à partir d'un décompte sommaire et forcément incomplet qu'une centaine de témoins paléo-babyloniens (la plupart étant issus de la période de la première dynastie de Babylone : 1800-1600 av. n. è.) nous sont parvenus. Dans les décombres de l'école d'un quartier d'habitations au centre de Nippur, 18 manuscrits de cette composition ont été retrouvés, ce qui illustre le fait qu'elle faisait partie des devoirs obligés des apprentis scribes en ce lieu vers 1700 (l'Hymne B à Innana, qui sort du lot, compte 36 exemplaires). Des témoins ont été découverts à Kiš, à Meturan dans la Diyala et à Suse soit à la périphérie de la Mésopotamie. En outre, une source indirecte mais très importante doit être prise en compte. Le titre de l'œuvre figure dans nombre de «catalogues » littéraires qui se rapportent presque exclusivement au corpus scolaire sumérien. Ces documents d'apparence modeste proviennent de Nippur, ville qui a livré la majorité des exemplaires d'Innana et Ebih connus jusqu'à ce jour, d'Ur et de Sippar. Trois autres « catalogues », dont un fameux conservé au musée du Louvre, sont de provenance inconnue. L'exemplaire de l'université de St. Andrews est d'après sa cursive le plus tardif des «catalogues » de cette série (fin de l'époque paléo-babylonienne). Celui-ci débute par exemple ainsi (les titres majoritairement en sumérien sont énumérés les uns au-dessus des autres) :

Maîtresse au cœur fort (= Innana $C$ ), Maîtresse aux dangereux pouvoirs (Innana et Ebih), Maîtresse aux innombrables pouvoirs (cosmiques) (=Innana B), An et Uraš (non identifié), Reine, que le grand An... (= Ninisina E), etc.

Ces listes recensent des hymnes divins et royaux, des poèmes narratifs, des débats et des proverbes ou autres textes de sagesse. Les lettres littéraires (un corpus scolaire d'environ 70 titres) ou les compositions liturgiques sont traitées généralement à part (sauf dans l'exemplaire de St. Andrews qui recense 12 lettres). Comme ce groupe documentaire est à l'évidence hétérogène, ses fonctions sont très débattues : il pourrait s'agir d'inventaires de tablettes (raisonnés ou non) ou de catalogues virtuels, - dans quelques cas, ce ne seraient que de simples mémorandums d'œuvres à connaître, voire l'expression d'un programme éducatif (curriculum).

L'hypothèse de l'inventaire de documents archivés a pour elle plusieurs cas dans lesquels il est explicitement question soit de rangement (dans des paniers), soit d'exemplaires matériels (compte du nombre de tablettes $d u b$ ou im-gid ${ }_{2}-d a$ ). La 
grande irrégularité des énumérations et le fait que les titres non encore identifiés ne soient pas rares vont aussi dans ce sens. Le seul intérêt qu'on peut trouver a priori à de telles listes est d'ordre comptable ou archivistique : elles devaient permettre une (future) vérification ou consultation. La motivation de l'opération reste cependant tout à fait énigmatique, car la possibilité d'un projet de conservation à long terme des documents inventoriés et de leur transmission paraît exclue. La notion de bibliothèque parfois présente dans la littérature scientifique ou secondaire est anachronique pour le début du deuxième millénaire, même si des fonds de textes littéraires ont dû exister, composés par exemple d'œuvres liturgiques, " outils » des praticiens du culte. Quel que soit le genre textuel, toute tablette devenue " inutile » était mise au rebut. De ce fait, leur « durée de vie » était variable. Il paraît superflu de rappeler que les tablettes littéraires ne se vendaient pas et qu'il n'y avait pas de librairie, pas plus qu'il n'y avait de bibliothèque au sens strict du terme. La preuve fournie par ces « catalogues » de l'existence d'un regroupement volontaire des documents écrits par « genre » et d'une conservation au moins temporaire est cependant considérable du point de vue de l'histoire littéraire puisqu'elle indique que l'objet-texte gardait une certaine valeur après sa fabrication et qu'il était intégré à une " série ». Le regroupement des titres d'œuvres constitue dès lors ce qui pourrait être considéré comme une bibliothèque virtuelle. Ce serait le témoignage d'une littérature orale dont la trace écrite ne constituerait qu'un produit dérivé (Delnero). Le cunéiforme était un support transitoire et circonstanciel. Tout au plus, les tablettes pourraient-elles servir d'aide-mémoire (fonction illustrée par un des nouveaux exemplaires d'Innana et Ebih de la collection Schøyen où n'est écrit que le début de chaque vers) ou de modèles (la circulation de lots de textes d'un centre à un autre est avérée).

Les œuvres pouvaient être regroupées par thème : ainsi dans le "catalogue " de la collection de l'université de St. Andrews, Innana et Ebih est associée à deux hymnes à Innana. Dans les trois inventaires dont le classement évoque le plus sûrement un programme scolaire, cette composition occupe la huitième place entre le Voyage d'Enki à Nippur et l'Hymne A à Nungal (cette succession paraît être purement conventionnelle). Un de ces «catalogues » provient de Nippur, un deuxième, celui du Louvre, est d'origine inconnue (Larsa est une possibilité). Le troisième manuscrit dont il n'existe qu'une translittération de J. van Dijk basée sur une autographie non publiée de F. W. Geers proviendrait de Sippar. Ces témoins prouvent qu'un classement standard a été largement diffusé en Babylonie au début du deuxième millénaire. Il ne serait pas étonnant que le modèle provienne de Nippur et Isin quoi que nous sommes peut-être victimes de la propagande faites par les maîtres de Nippur sur l'excellence de leur formation.

Dans un texte administratif typique, le chiffre est mis en évidence par la disposition du texte qui fait ressortir cette donnée. Le petit groupe d'inventaires des lamentations-balag entre assez bien dans cette catégorie. Le nombre de tablettes par titre y est toujours indiqué, tandis que les tablettes d'extraits im-gid ${ }_{2}$ - $d a$ sont recensées à part (les tablettes sont donc triées par type). Or, cette caractéristique est absente de la majorité des autres «catalogues » qui se contentent de ranger les titres, ligne à ligne avec, dans un cas ou deux, une subdivision toutes les dix lignes (la marque de contrôle PAP s'y ajoute). La présence éventuelle du clou oblique « 10 » en marge 
témoigne d'un décompte des lignes à la manière des textes littéraires eux-mêmes. Un des catalogues d'Ur (UET VI 123) donne un total non des lignes mais des œuvres recensées (« total 67 [titres] », un titre pouvant se rapporter à plusieurs œuvres, ex. : «10 [compositions ayant pour incipit] Le roi »). Plus important que le nombre de tablettes produites (même si ce critère peut être pris en compte) est la description du corpus des œuvres copiées. Une liste de «titres », la plupart non identifiés, indique explicitement que seulement quelques-unes des œuvres recensées ont été recopiées intégralement. L'implication d'une telle information est difficile à établir, mais pour M.-C. Ludwig, qui a édité cette tablette atypique du British Museum, ce serait l'indice que le document en question était conçu comme un bilan.

L'important est que toutes ces informations montrent qu'Innana et Ebih était une œuvre quasiment obligée pour un élève ayant atteint un niveau élevé de la formation scribale (fait souligné par l'exemplaire de la collection Schøyen MS 3176/1 K. Volk et J. Matuszak doivent prochainement le publier - qui l'associe à d'autres œuvres notables du répertoire). Dès lors, les images et les idées qui y sont véhiculées ont été largement diffusées dans le monde mésopotamien et ont contribué à façonner les esprits. Il convient d'extraire ce document de ce contexte strictement scolaire qui l'a préservé pour tenter de saisir plus largement le message qu'il adresse à la société babylonienne.

Innana et Ebih quoique très étudiée attend toujours une édition « définitive ». En attendant, une partie du matériel épigraphique est facilement accessible sur le site du CDLI même s'il n'est pas complet. On y trouve ainsi de nombreux clichés des tablettes de Philadelphie (musée de l'université de Pennsylvanie), du musée du Louvre, du British Museum (pour les tablettes d'Ur), etc., sans compter les remarquables exemplaires encore inédits de la collection Schøyen. Ces données permettent de reconstituer une tablature partielle bien sûr basée sur l'excellent travail éditorial préexistant (édition de P. Attinger, à compléter par les articles de M. Jaques, P. Delnero, etc.).

Une des premières difficultés qui se présente lorsque l'on souhaite étudier ce genre de documents est le fait qu'aucun exemplaire ne donne de version complète du texte. Le poème nous est parvenu soit sous forme de versions à l'origine intégrales (grandes tablettes à 4 colonnes ou prismes) soit sous la forme d'extraits. Dans le premier cas, aucun des exemplaires à notre disposition n'est intact. Le témoin le plus remarquable dont nous nous sommes servis est UM 29-16-32. Malheureusement la face étant déchiquetée, seul son revers était vraiment exploitable. Dans le second cas, les extraits qui nous sont parvenus ne forment aucune série continue qui permettrait de lire d'un bout à l'autre le texte dans une seule version, c'est-à-dire résultant de la main d'un même scribe. De telles séries sont attestées par ailleurs comme l'indique ce colophon de UET VI 10 : «Troisième extrait; partie finale : Du grand Ciel vers la grande Terre (incipit de la Descente d'Innana aux Enfers; im-gid ${ }_{2}$-da 3-kam za til-la, an-gal-ta ki-gal-š $e_{3}$ ) ». Plusieurs extraits d'Ur (UET VI 11, 13, 15, 17), caractérisés par leur format paysage (le texte part de la gauche du petit côté) ont sûrement été écrits par le jeune scribe, Ibni-Sîn (celui-ci s'identifie uniquement sur le premier extrait). Malheureusement, il nous manque les parties contenant les lignes 67 à 95 et 120 à 150 (peut-être représentées toutefois par le fragment UET VI 16). 
Cette situation fait que le texte complet d'Innana et Ebih est seulement une reconstitution moderne (on parle de texte composite). En outre, il est bien sûr impossible de le lire d'un bout à l'autre en cunéiforme dans une version intégrale commode. L'établissement d'un texte cunéiforme composite est une étape intermédiaire entre la translittération et le document original (hermétique à un œil non exercé) qu'il soit sous forme d'autographie ou mieux en photographie numérique. La copie traditionnelle réalisée à la main et assistée d'un logiciel de création graphique pour réaliser le montage permet de constituer aisément un modèle qui sera sujet à critique tout comme le texte composite qui nécessairement doit effectuer un choix entre les variantes.

Le projet paléo-babylonien se heurte cependant au problème de la variété des graphies et des ductus. La forme de l'écriture évolue fortement entre 1900 et 1600 qui correspond à la phase la mieux documentée. De plus, au XVIII ${ }^{\mathrm{e}}$ siècle, notamment à Nippur et à Ur, au moins deux formes d'écriture se côtoient, l'une scolaire qui retient des caractéristiques graphiques anciennes et l'autre simplifiée qui représente la cursive (c'est l'écriture des « catalogues » par exemple). Ces deux niveaux d'écriture se rencontrent parfois dans les mêmes textes. Les dessins des signes cunéiformes sont toujours reproduits d'après les documents photographiques (par conséquent, les tablettes d'Istanbul dont nous n'avons que des copies n'ont pas pu être prises en compte ce qui n'a guère été gênant vu la masse de textes disponibles). L'intérêt de cette version composite établie à partir de graphies authentiques tirées des manuscrits d'Innana et Ebih et fidèlement imitées, est de confronter l'étudiant, le lecteur, à différentes façons d'écrire un même signe.

Une autre difficulté est, plus que l'établissement du texte ou l'analyse grammaticale, celle de l'étude lexicale et de la traduction (cette dernière est facilitée par la diffusion de plusieurs glossaires récents). Une simple comparaison des traductions disponibles établies par les maîtres de la sumérologie (en français, anglais et allemand) laisse entrevoir de considérables et a priori étonnantes divergences. Quant à nous, si nous nous sommes livrés à cet exercice particulièrement stimulant dans le cadre interactif du cours, c'était dans le but d'accéder au cœur du texte avec nos outils, pour ainsi en dégager notre propre interprétation. L'objectif était d'en comprendre avec le plus de finesse possible la grammaire, la poétique et le message idéologique.

Nous nous contenterons de donner ici in extenso le début du poème tel que nous l'avons compris (quelques remarques préliminaires : 1. 18: la majorité des manuscrits donnent $\mathrm{TUG}_{2}-\mathrm{TUG}_{2}$-ra, nous n'avons rencontré LAGAB.LAGAB que dans un seul témoin de Nippur; 1.67 : la présence de la copule $a_{3}$ nous a laissé un doute sur le sens exact de cette ligne, l'interprétation de J. Bottéro et N. Kramer a été abandonnée mais demeure à notre avis intéressante, ils spéculent que an-bar-ra serait le nom d'une arme divine; en s'en inspirant, sachant que an-bar peut signifier « fer», on pourrait comprendre «En-fer, qui est une hache »; concernant la difficile 1. 70 de nombreuses variantes sont apparues : $m u-B U$ (Nippur et Ur), giš- $B U$ (Nippur : $\mathrm{N} 1357) ; m u-B U-u r_{2}$ (YRL SC 1826); $\hat{g} i \check{s}$-šir 3 (MS 3340), lesquelles invalident la lecture souvent retenue ${ }^{* *} m u-b u-u m$; la présence d'un terme Emesal ( $\hat{g} i \check{s}=m u$ ) dans le discours de la déesse est à signaler; même si le passage garde son obscurité, nous 
faisons néanmoins un parallèle avec Ur-Ninurta A, 1. 23 : sibir-a-ni ki-bal he ${ }_{2}$-engam-e «Qu'il soumette sous sa houlette les pays rebelles! »; grâce à une idée d'une auditrice nous proposons de lire à la $1.77 \mathrm{izi}$ gu $u_{7}$ 《 dévorer par le feu » (une forme non conjuguée paraît en effet préférable); à la 1.81 : le verbe n'est pas $̌ s u d u b_{2}$ mais $s u^{*}$ $d u b_{2}$ «trembler de peur» (cf. CBS 13977, BPOA 923 et copie ci-dessous); le verbe $s a \hat{g} d u b_{2}$ soit litt. " secouer la tête " ou « hocher la tête " pourrait signifier " regarder avec colère » (akk. nekelmû) d'après la comparaison avec le faucon qui est le thème des 1. 80-84 : Innana (ou le roi) identifiée au rapace prend son envol pour repérer ses proies dans son territoire de chasse, ce qui provoque la panique chez les ennemis, ici représentés sous la forme de serpents; 1.81 peut être rapprochée de Šulgi A 45 : « comme un aigle qui a porté le regard sur sa montagne, je me suis élancé dans la

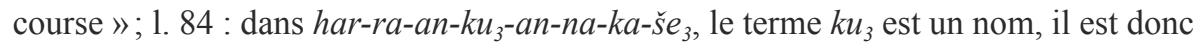
bien question d'un chemin [pavé] d'argent) :

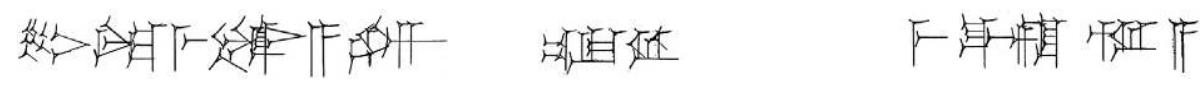

Maîtresse aux pouvoirs terribles, porteuse de l'aura, qui dompte les grands pouvoirs
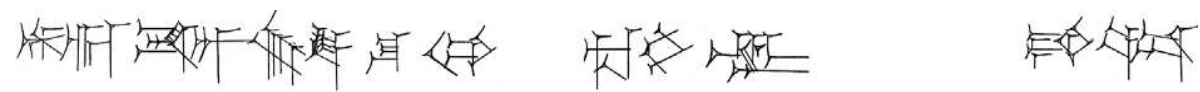

${ }^{2}$ Innana qui manie à la perfection l’(arme) Ankar, éclaboussée du sang du combat
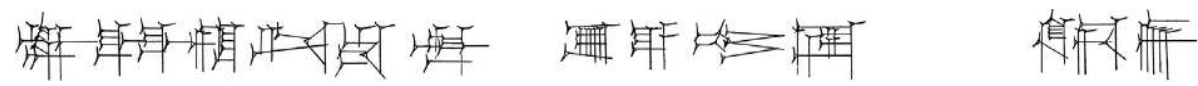

Sautant allègrement dans les grands combats, le bouclier laissé à terre
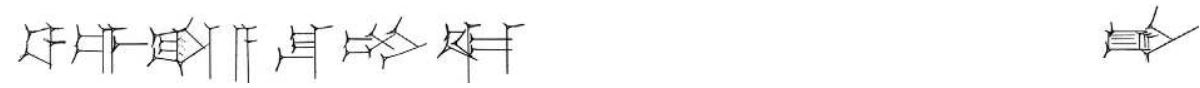

${ }^{4}$ Parée de tempêtes et d'ouragans

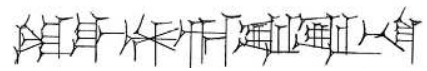

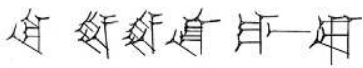

Grande Reine, Innana experte en stratagèmes de guerre.

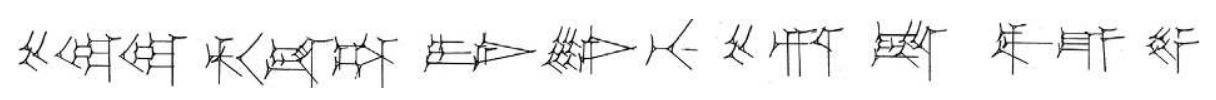

${ }^{6}$ Destructrice des pays, décoches-tu une flèche, le coup (fatal) est asséné sur le pays (visé).

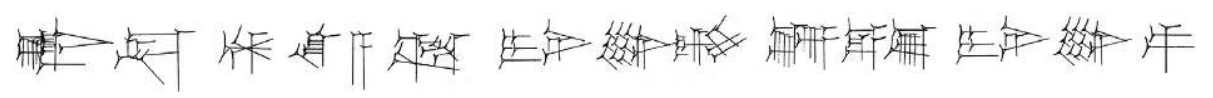

(Aussitôt que) tu émets un cri dans l'univers, tel un fauve, tu as frappé de terreur les gens.

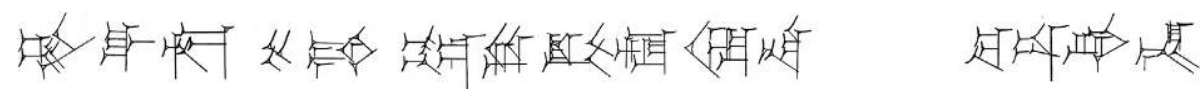

${ }^{8}$ Tel un massif taureau sauvage, tu triomphes du pays hostile. 


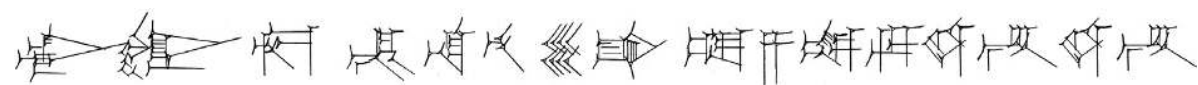

Tel un fauve féroce, tu glaces de ta colère (l'ardeur de) l'impétueux et du désobéissant.

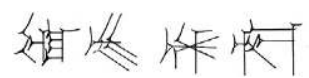

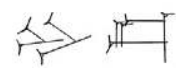

${ }^{10} \mathrm{Ma}$ reine, (le fait) que tu as grandi comme le ciel,

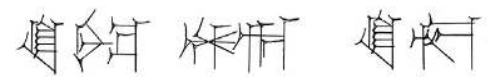
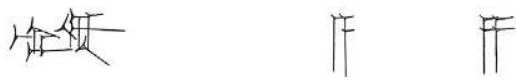

Jeune femme Innana, que tu es devenue aussi grande que la terre,

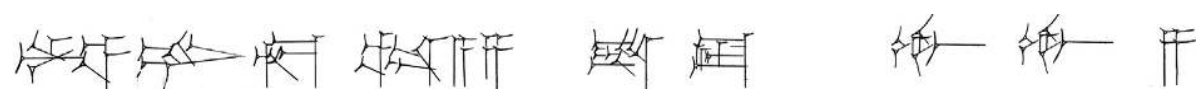

${ }^{12}$ Que tu te manifestes comme Utu le roi, que tu déploies de grandes ailes,

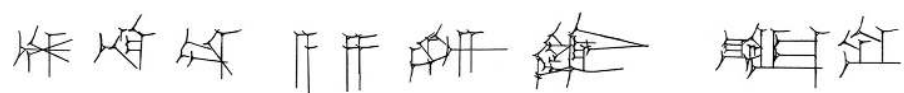

Que tu parcours le ciel, que tu es porteuse d'une aura redoutable,

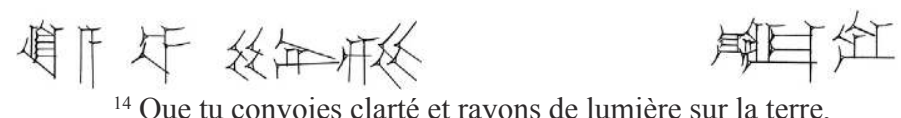

\#

${ }^{14}$ Que tu convoies clarté et rayons de lumière sur la terre,

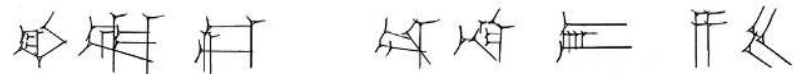

Que parcourant la montagne tu fais apparaître un « filet » bleu-étincelant,
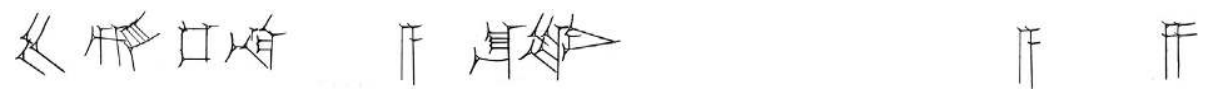

${ }^{16}$ Que tu te baignes dans la montagne translucide,

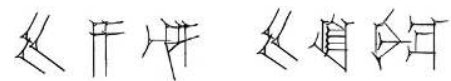

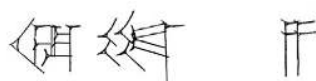

Que (toujours) tu (re)nais Montagne et Jeune Femme dans la montagne cristaline,

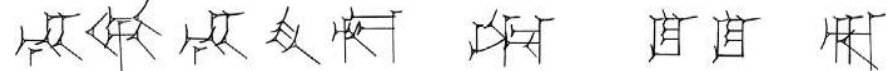

${ }^{18}$ Que tu portes sur toi une masse d'arme à la façon (des anciens dieux) Enul et Enšar,

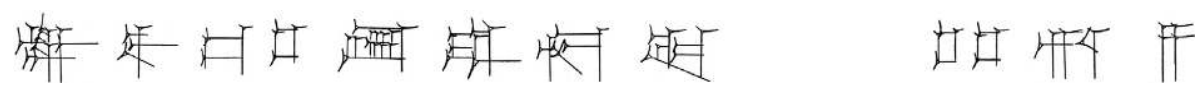

Que tu fais rouler, dans de ces combats, les têtes comme des boules faisant du ravage (= un jeu de quilles ?),
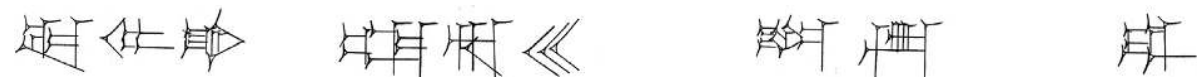

${ }^{20}$ (Cela) les Têtes-noires l'ont chanté, 


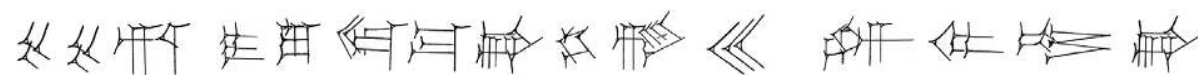

Tous les pays entonnent mélodieusement en cet honneur leurs ilulam.

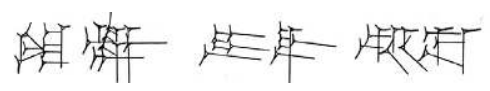

${ }^{22}$ Reine du combat, fille aînée de Suen

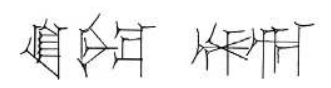

Je veux te louer jeune Innana!

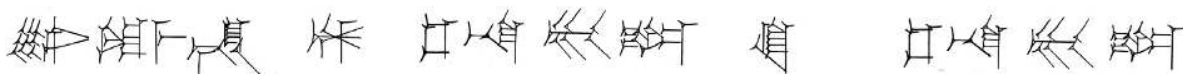

${ }^{24}$ - Moi qui suis la Maîtresse, lorsque je faisais le tour du ciel, lorsque je faisais le tour de la terre,

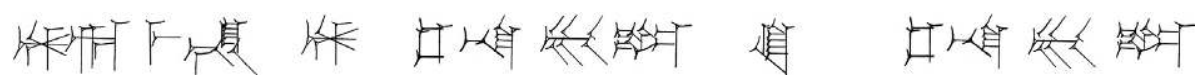

Moi qui suis Innana, lorsque je faisais le tour du ciel, lorsque je faisais le tour de la terre,

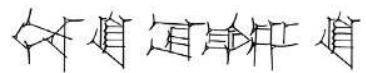

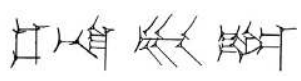

${ }^{26}$ Lorsque je faisais le tour de l'Élam et du Subartu,

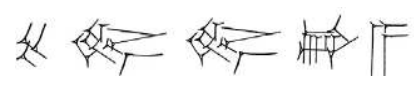

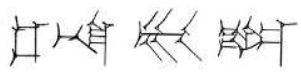

Lorsque je faisais le tour du pays des Lulubu,

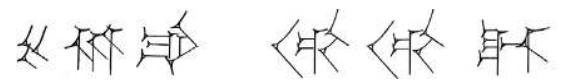

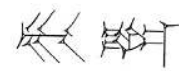

${ }^{28}$ Lorsque menant une incursion aux fins fonds de la Montagne,

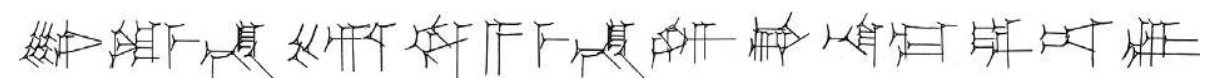

Moi qui suis la Maîtresse, quand je me suis approchée de la montagne, il ne m'a pas craint.

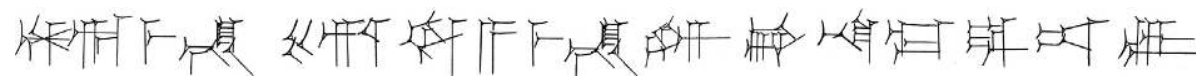

${ }^{30}$ Moi qui suis Innana, quand je me suis approchée de la montagne, il ne m'a pas craint.

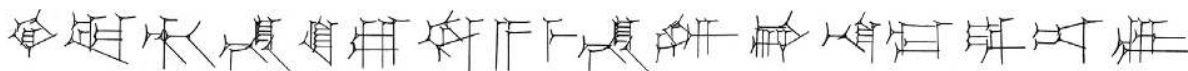

Le mont de l'Ebih ne m'a pas craint, moi qui m'approchait.
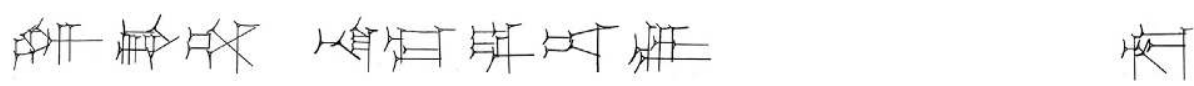

${ }^{32}$ Comme il ne m'a craint en aucune manière, 


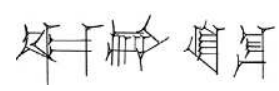

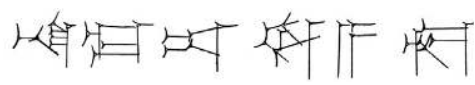

Comme il ne s'est pas prosterné devant moi,

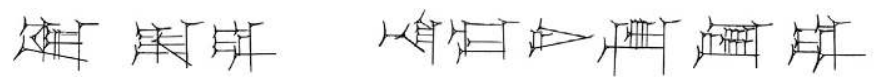

${ }^{34}$ Comme il n'a pas balayé de sa barbe la poussière devant moi,

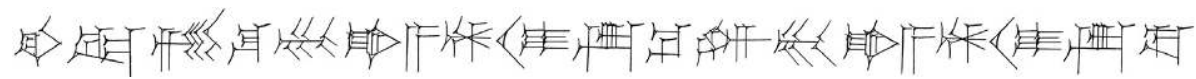

Je veux remplir mes mains de $<$ cet $>$ orgueilleux mont ! Je veux qu'il goûte à ma terreur !

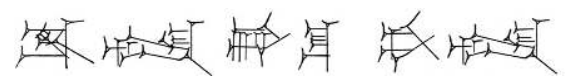

${ }^{36} \mathrm{Je}$ vais dresser contre sa puissante force un taureau (aussi) puissant,

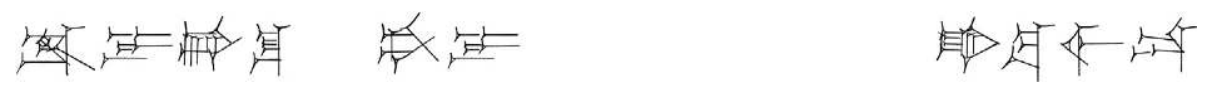

Et contre sa force impétueuse dresser un taureau (aussi) impétueux (= jeune)!

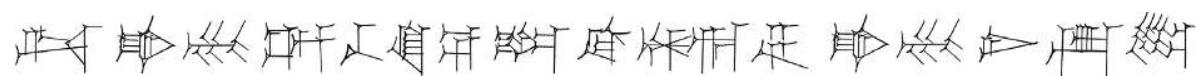

${ }^{38} \mathrm{Je}$ veux y galoper et y sauter à la corde sacrée !

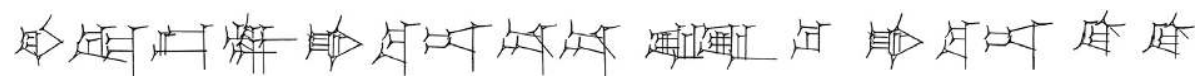
Je veux mener la guerre totale sur le mont, y déchaîner les combats.

还脏道等作

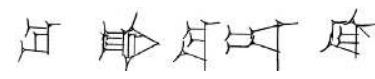

${ }^{40} \mathrm{Je}$ vais préparer les flèches dans le carquois,

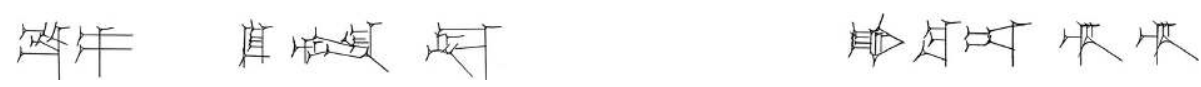

« Tresser » une fronde (dure) comme de la corde !

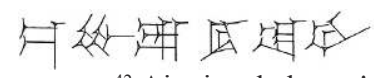

${ }^{42}$ Aiguiser la lance !

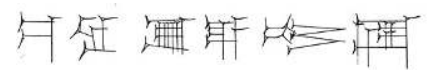

Préparer ce qu'il faut de propulseurs et d'armes,

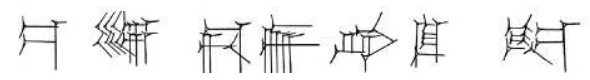

${ }^{44}$ Mettre le feu à ses forêts qui le jouxtent,

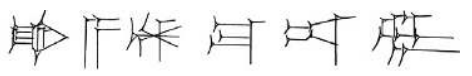

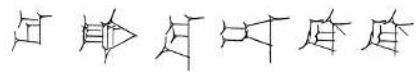




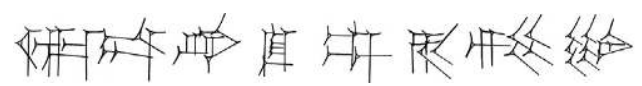

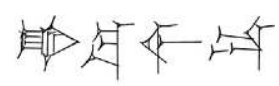

(Et) je vais actionner la cognée contre les méchants qui l'habitent!

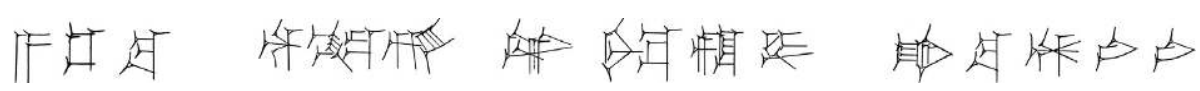
${ }^{46}$ (Jusque) dans ses réservoirs d'eau, je donnerai beaucoup de travail au (dieu) Gibil le purificateur!

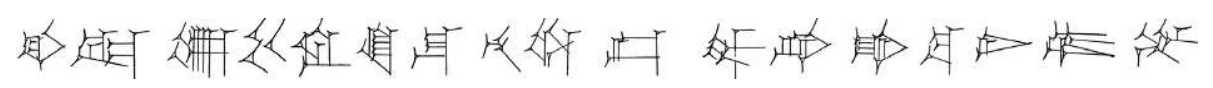

Son effroi je vais le répandre même jusque dans l'inaccessible montagne d'Aratta!

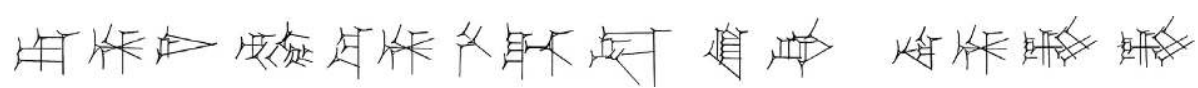

${ }^{48}$ Telle une ville qu'An aurait maudite, jamais (Ebih) ne pourra plus être restauré.

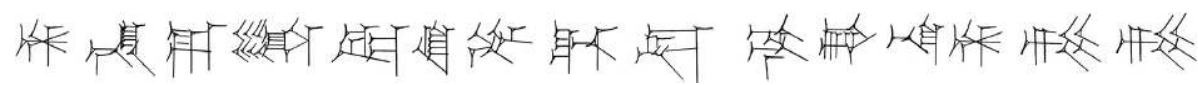

Comme si Enlil s'était mis en colère (contre lui), jamais plus il ne relèvera la nuque.

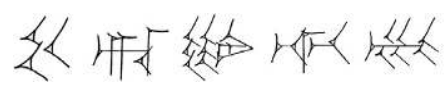

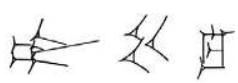

${ }^{50} \mathrm{Il}$ aurait mieux valu que mes actions (passées) soient remémorées à la montagne,

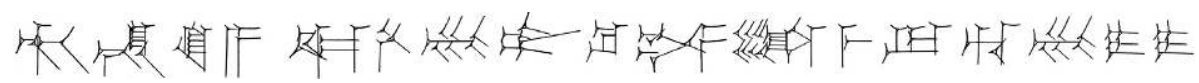

Et que ma gloire résonne et soit célébrée dans l'Ebih!

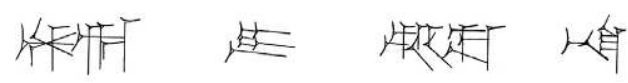

${ }^{52}$ Innana fille de Su'en

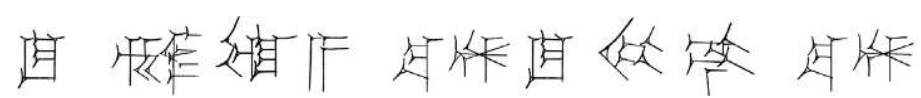

Se revêtit de son habit royal, mit son manteau tout en splendeur.

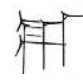

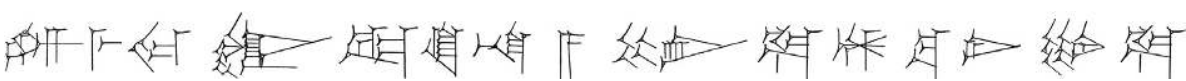

${ }^{54}$ Elle s'illumina le front d'un rayonnement terrible.

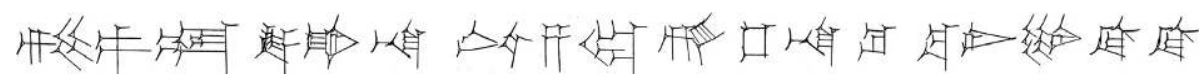

(Et) arrangea autour de son cou sacré (un collier) à (perles de) cornaline en forme de rosaces.

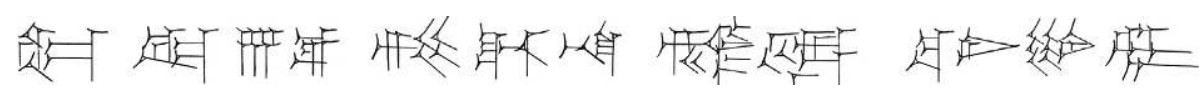

${ }^{56}$ Elle mania avec virilité à sa droite la masse d'arme à 7 pointes. 


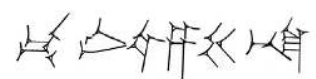

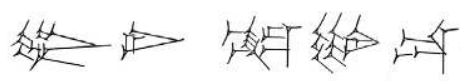

(Puis) elle posa son pied sur l'échelle de lazulite.

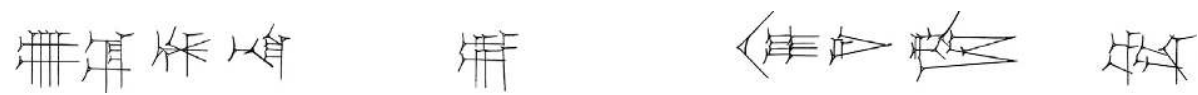

${ }^{58}$ (Celle-ci) la fit s'élever en majesté dans le crépuscule,

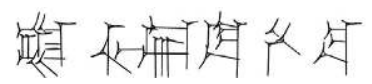

链绘战

Sur cette voie, elle ouvrit la porte des merveilles.

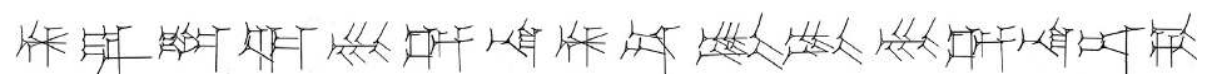

${ }^{60}$ Ayant apporté à An une offrande, elle lui adressa une prière.

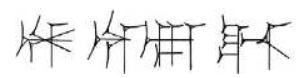

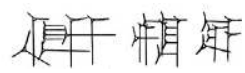

An, fort content d'Innana,

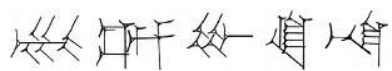

金年庐

${ }^{62}$ L'attira et elle prit place

造年年

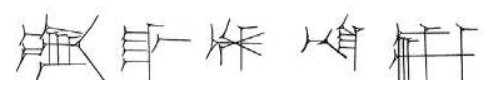

(La voilà qui) occupait la grande chapelle du ciel.

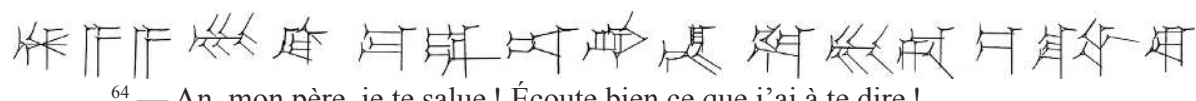
${ }^{64}$ - An, mon père, je te salue ! Écoute bien ce que j'ai à te dire !

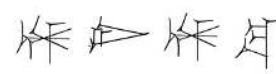

奸 从स

但事

An a-t-il vraiment imposé dans ce ciel mon aura de terreur?

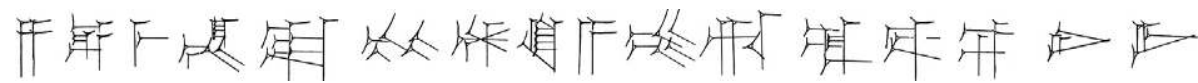

${ }^{66}$ C'est toi même qui n'as pas permis que dans l'univers ma parole trouve un rival.

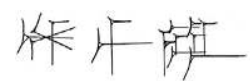

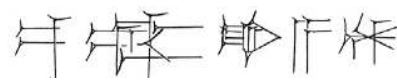

À l'extrémité du ciel (où tu es), la hache en propre,

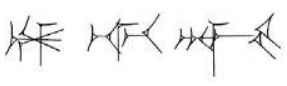

${ }^{68}$ (Les insignes de pouvoir) antibal et mansium,

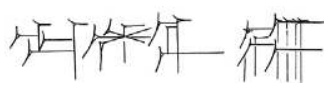




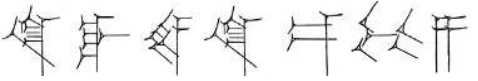

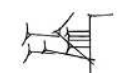

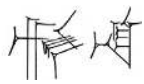

Établir le socle (et) la base solide du trône,
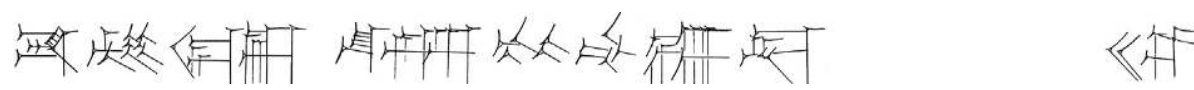

${ }^{70}$ Prendre en main l'arme digne du chef suprême, faire ployer (les rebelles) par le sceptre comme avec le poing,

\section{䉼十年迤}

स स

Avoir le contrôle d'un attelage de 6 (destriers),

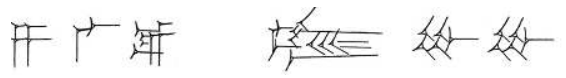

${ }^{72}$ Lancer à pleine vitesse l'attelage de 4 (destriers),

政太每

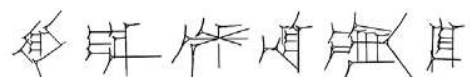

Mener des raids meurtriers et conduire des campagnes (militaires) jusqu'au bout,

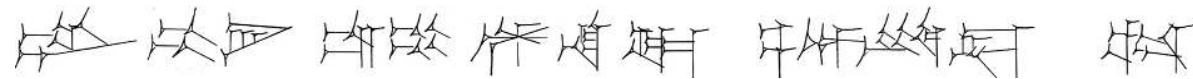

${ }^{74}$ Surgissant au devant de leur roi comme la lune au milieu de la poussière soulevée dans le ciel,

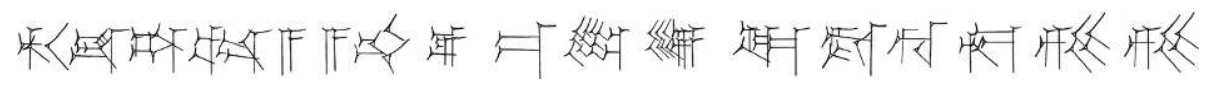

Et tout en décochant des flèches, arracher, à l'égal de la voracité des criquets, vergers et forêts pour en faire des champs (nus),
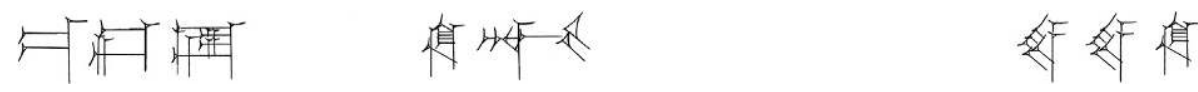

${ }^{76}$ Ratisser le territoire rebelle

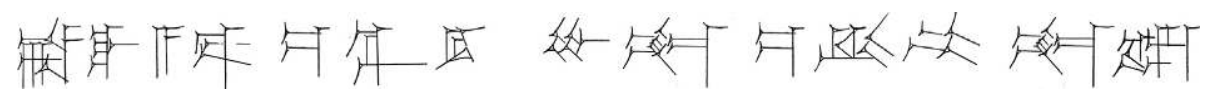

Retirer à sa grand'porte les verroux et mettre le feu à ses vantaux,

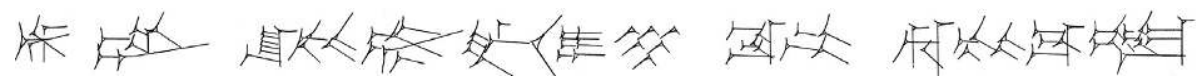

${ }^{78}$ An seigneur, tu as, c'est un fait, remis entre mes mains (ces choses) et tu m'as donné ces missions!

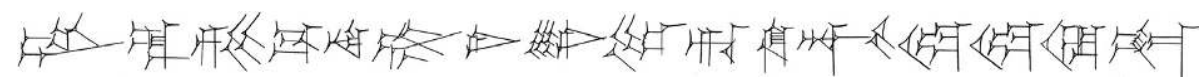

Puisses-tu me faire retourner à la droite du roi pour anéantir le pays rebelle ! 


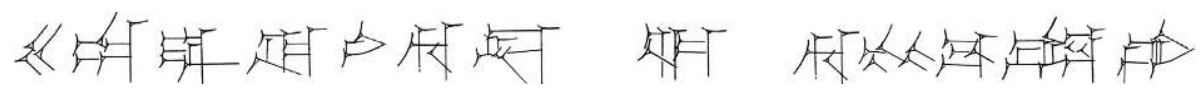

${ }^{80}$ Pour qu'avec moi il foudroie du regard le fond de la montagne comme le faucon.
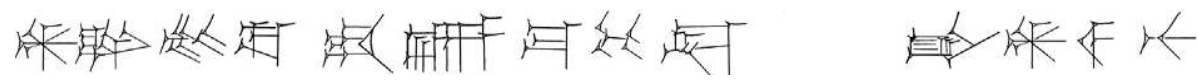

An seigneur, je veux étendre ta renommée comme un fil au-delà des limites du Pays.

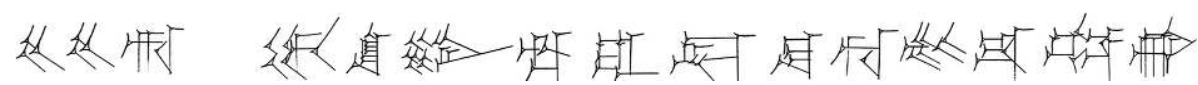

${ }^{82}$ Puissent trembler tous les pays devant moi comme un serpent (apeuré) dans (son) trou.

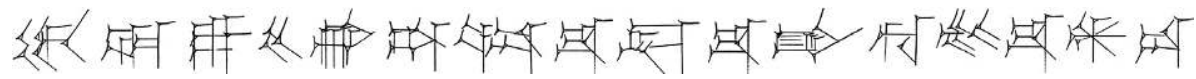

Puissent-ils se disperser devant moi comme le serpent saĝ.kal qui quitterait sa montagne.

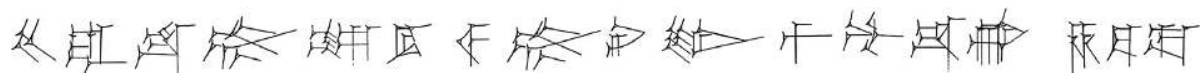

${ }^{84}$ J'ai « posé le bras » sur la contrée (pour la mesurer), je l'ai examinée et j'en connais l'étendue.

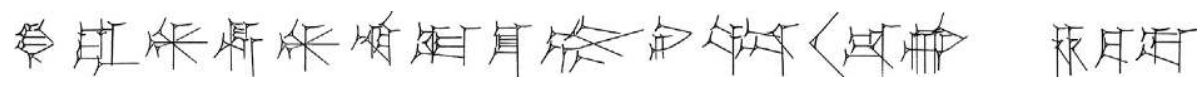

Je me suis élevée sur la voie argentée du ciel et j'ai ainsi appris quelle en était la profondeur.

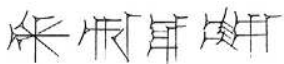

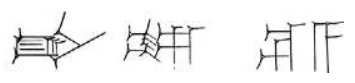

${ }^{86} \mathrm{Je}$ veux surpasser les dieux.

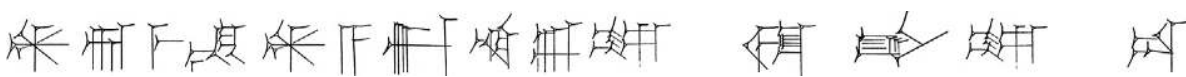

Moi Innana, je veux ouvrir la marche pour les Anuna.

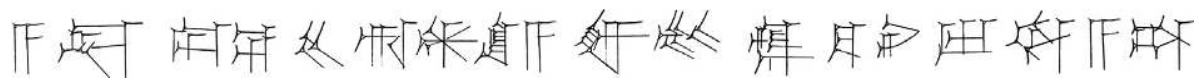

${ }^{88}$ Comment se fait-il donc, que moi, une montagne au sein de l'univers a cessé de me craindre,

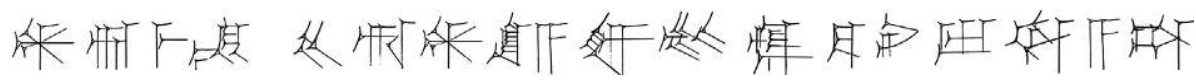

Que moi qui suis Innana, une montagne au sein de l'univers a cessé de me craindre,

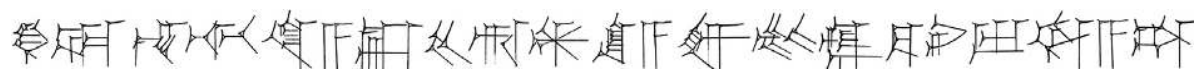

${ }^{90}$ Que le mont Ebih, une montagne, au sein de l'univers, a cessé de me craindre? 
La date de création et l'origine d'Innana et Ebih sont inconnues. Le préambule fait allusion à des hymnes ou des récits sur Innana qui étaient populaires. Les habitants de Sumer la vénéraient en tant que Vénus et déesse guerrière, réputée pour sa férocité. Le présent poème fera le récit d'un nouvel exploit divin. Cette action pourrait se référer vaguement à un épisode militaire remontant à l'époque d'Akkad. Le caractère mythologique et idéologique est plus manifeste que ce fil historique bien ténu mais très plausible. Les liens d'Innana avec la royauté sont étroits : sa défense est sa mission sacrée, aussi s'apprête-t-elle à de nouveau épauler « le roi » (1. 79). Quelques lignes plus haut, il est fait allusion à l'affrontement avec un (autre) roi (1. 74) qui conduira à la destruction de sa cité rebelle (1. 75). Temps mythique et historique se superposent. Le discours d'Innana (1. 67-77) nous apprend qu'An en personne lui a confié la défense de la royauté qu'elle incarne aussi. Le dieu suprême lui a cédé les pleins pouvoirs de la guerre, ce qui prouve que l'entreprise de vengeance qu'elle veut mener est d'emblée légitime. Le propos est parfois obscur peut-être en raison de son caractère archaïque; il est aussi un peu en décalage avec le reste du poème parce qu'il n'a pas de rapport clair et direct avec le sujet principal (sauf la thématique très générale de la guerre) et qu'il y est question d'une hache donnée par An, une arme divine donc. Mais elle ne fait pas partie de la panoplie décrite dans le reste du texte où il est en particulier question de l'Ankara (1. 2), qui est probablement la massue évoquée 1. 56, ainsi que d'une hache-hazin (1. 45). La hache sišsilig était aussi celle que

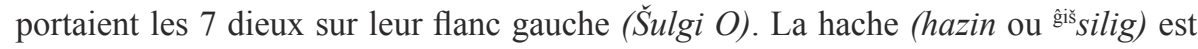
bien l'instrument adéquat pour abattre les arbres du pays adverse (thème répété aux 1. 45 et 75). Innana exprime enfin de manière imagée son programme expansionniste : il consistera à détruire le paysage de l'ennemi en abattant ses arbres et ses cités. La curieuse manière de décrire cela suggère que son intention est de transformer l'espace conquis en terres arables, afin de l'adapter au modèle d'exploitation économique « babylonien ». Une idée de colonisation est peut-être sous-jacente. Il y a par conséquent chez Innana, malgré son évidente hubris, un véritable projet civilisateur.

Ce sujet avait occupé le second semestre, tandis qu'au premier nous nous étions penchés sur un dossier de lettres en akkadien (certaines récemment éditées et d'autres inédites) provenant peut-être d'une cité nommée Rahabum et datées du règne de Sumu-El ( $\mathrm{XIX}^{\mathrm{e}}$ siècle av. $\mathrm{n}$. ère). On a tenté de remettre en ordre chronologique les lettres (elles n'étaient pas datées) ce qui nous a permis de reconstituer en partie un épisode historique vu depuis une ville frontalière du royaume de Larsa, ainsi que d'entrevoir des aspects de l'administration locale.

\section{Références bibliographiques principales}

P. Attinger, «Inana et Ebih », ZA, 88 (1998), p. 164-195.

J. Bottéro et S. N. Kramer, Lorsque les dieux faisaient l'homme. Mythologie mésopotamienne, Paris, 1983 (rééd. 1993).

D. Charpin, Le Clergé d'Ur au siècle d'Hammurabi, Genève, Paris, 1986.

P. Delnero, " "Inana and Ebih" and the Scribal Tradition », dans G. Frame et alii (éd.), A Common Cultural Heritage: Studies on Mesopotamia and the Biblical World in Honor of Barry L. Eichler, Bethesda, 2011, p. 123-149. 
P. Delnero, « Sumerian Literary Catalogues and the Scribal Curriculum », ZA, 100 (2010), p. 32-55.

M. Jaques, «Inanna et Ebih : nouveaux textes et remarques sur le vocabulaire du combat et de la victoire », ZA, 94 (2004), p. 202-225.

M.-C. Ludwig, "BM 96740 - eine altbabylonische Liste von Textanfängen », dans C. Mittermayer et S. Eklin (éd.), mu-ni $u_{4} u l-l i_{2}-a-a \check{s} \hat{g} a_{2}-\hat{g} a_{2}-d e_{3}$. Altorientalische Studien zu Ehren von Pascal Attinger, Fribourg, Göttingen, 2012 (OBO 265), p. 201-213

J. Peterson, The literary sumerian of Old Babylonian Ur: UET 6/1-3 in transliteration and translation with select commentary, 2016, sur Academia.edu.

E. Robson, « The Tablet House: A Scribal School in Old Babylonian Nippur », RA, 95 (2001), p. 39-66.

S. Tinney, « On the Curricular Setting of Sumerian Literature », Iraq, 41 (1999), p. 159-172.

K. Volk (éd.), Erzählungen aus dem Land Sumer, Wiesbaden, 2015.

A. Zgoll, «Ebeh und andere Gebirge in der politischen Landschaft der Akkadzeit », dans L. Milano et al. (éd.), Landscapes: Territories, Frontiers and Horizons in the Ancient Near East, II. Geography and Cultural Landscapes, Padoue, 2000 (Rencontre assyriologique internationale, 44), p. 83-90. 